\title{
Fast Two-Dimensional Direction-of-Arrival Estimation of Multiple Signals in Coprime Planar Array
}

\author{
Haiyun Xu (i), Yankui Zhang $\left(\mathbb{D}\right.$, Bin Ba $\mathbb{D}^{D}$, Daming Wang, and Peng Han \\ National Digital System Engineering and Technological Research R \& D Center, Zhengzhou 450001, China \\ Correspondence should be addressed to Yankui Zhang; zhang_yk_2018@foxmail.com
}

Received 24 October 2018; Accepted 14 April 2019; Published 27 June 2019

Academic Editor: Jaime Lloret

Copyright (C) 2019 Haiyun Xu et al. This is an open access article distributed under the Creative Commons Attribution License, which permits unrestricted use, distribution, and reproduction in any medium, provided the original work is properly cited.

\begin{abstract}
Two-dimensional direction-of-arrival (DOA) estimation in coprime planar array involves problems that the complexity of spectral peak search is huge and the noncircular feature of signals is not considered. Considering that unitary estimating signal parameters via rotational invariance techniques (Unitary-ESPRIT) is a low complexity subspace algorithm, an approach to estimate DOA fast for multiple signals is proposed in this paper. We first apply Unitary-ESPRIT to solve one possible value of each signal. Given the relationship between the ambiguous values and real values, we then have all possible values belonging to each subarray. Through finding the common values of two subarrays, we finally obtain the highly precise true DOAs. Moreover, when the signals are noncircular, we present an improved method using noncircular Unitary-ESPRIT, which is favorable in terms of accuracy and degree of freedom. Simulation results demonstrate the effectiveness of our proposed methods.
\end{abstract}

\section{Introduction}

Direction-of-arrival (DOA) estimation of multiple narrowband signals is a fundamental task in many applications such as radar [2], underwater acoustics [3, 4], indoor navigation [5, 6], and wireless communication [7]. The uniform rectangular arrays (URAs) [8] using subspace algorithms can obtain highresolution two-dimensional (2D) DOA estimations. In recent years, the coprime array [9-12] has become a focus, which consists of two uniform sparse arrays. Compared with URAs, the coprime planar array has a higher array aperture with the same number of sensors. And a partial spectrum search (PSS) method in coprime planar array is presented in $[13,14]$, which resolves the DOAs via finding the common peaks of two subarrays, but the spectral peak search costs much complexity, especially with a small searching step to acquire high accuracy. We have proposed a method in [1], which uses the covariance matrix of coprime planar array to estimate a new covariance matrix with matrix completion theory. This processing has much improved degree of freedom (DOF) bigger than the number of sensors, but the matrix completion costs much complexity and introduces the additional errors. Thus, when we want to secure a highly accurate estimation value with small complexity, this method may be not efficient enough.

Furthermore, the existing algorithms have just taken the independent narrowband signals into consideration. But sometimes the features of impinging signals can influence the performance. Considering that the general subspace methods cannot be applied to coherent signals, we have proposed an algorithm in [15] to resolve DOAs of both uncorrelated and coherent signals in coprime planar array. Moreover, there are many communication signals with ASK, BPSK, and UQPSK modulation [16, 17], which have the noncircular features, and some with QPSK and QAM modulation [18], which have the circular features. Independent noncircular signals, whose ellipse covariance matrix is nonzero, can expand the array aperture and enhance the accuracy $[19,20]$, while the circular signals cannot. However, the extended array aperture causes the complexity increased. As a result, it is meaningful that we additionally propose a low complexity method for noncircular signals, which can utilize the noncircular feature to realize fast DOA estimation in coprime planar array. An algorithm is presented for noncircular signals [21], but it can only have favorable performance when array aperture is not big. 


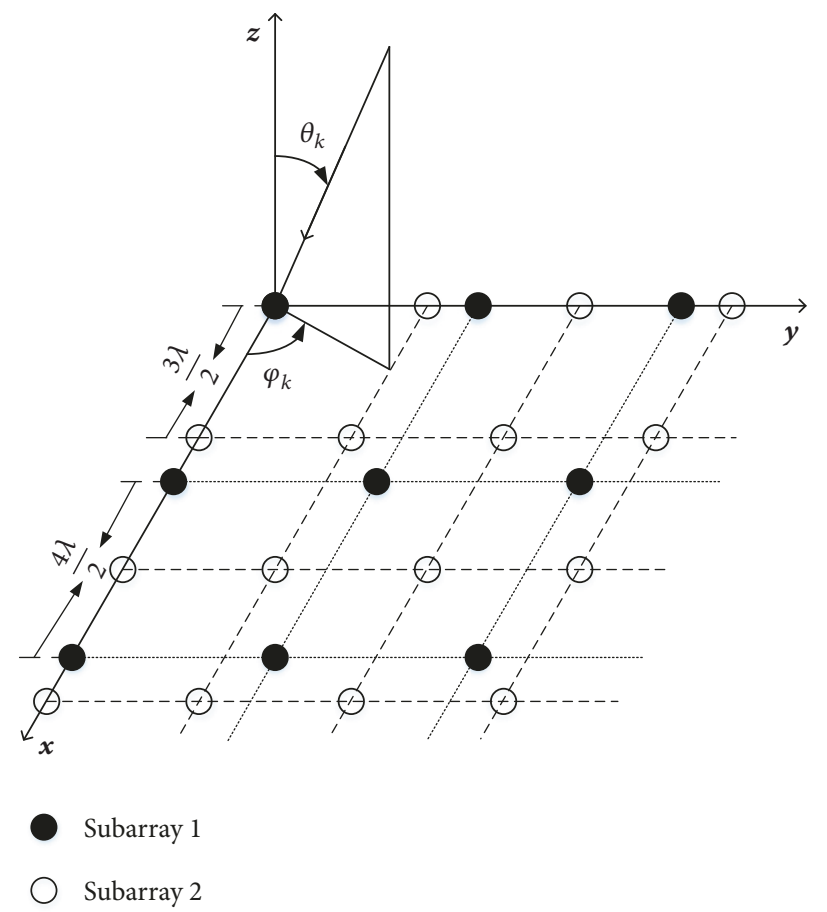

FIGURE 1: Geometry of coprime planar array [1] when $M_{1}=3$ and $M_{2}=4$.

In order to avoid the high complexity cost by the spectral searching and utilize the signals features, in this paper, a fast DOA estimation method in coprime array for multiple signals is proposed. We first obtain the received signal data and calculate the covariance matrix. Then, when signals are circular, we can apply unitary estimating signal parameters via rotational invariance techniques (Unitary-ESPRIT) [2225], a low complexity algorithm, to solve the closed-form solutions, which are possible estimated values. When the signals are noncircular, we make an improvement and present the noncircular Unitary-ESPRIT (NC-Unitary-ESPRIT) to solve the closed-form solutions. Through the relationship between the ambiguous values and real values, we can obtain all the possible values of each subarray. By finding the common values between two subarrays, the true estimated DOAs are obtained with high precision. Compared with PSS, both Unitary-ESPRIT and NC-Unitary-ESPRIT can reduce the complexity. Moreover, NC-Unitary-ESPRIT has much improved the accuracy and DOF with the noncircular feature.

The paper is organized as follows. We first present a brief signal model in Section 2. In Section 3, we explain our approach to estimate DOAs and show the steps of the proposed algorithm. The computational complexity and DOF analysis are presented in Section 4. In Section 5, we show the results of simulations. Finally, we summarize work in Section 6. Throughout this paper, $\mathbf{I}_{N}$ represents the $N$ dimensional unit array; $[\bullet]^{T}$ and $[\bullet]^{H}$ denote transpose, and Hermitian transpose, respectively; $\operatorname{diag}[\bullet]$ represents a vector transforming to a diagonal matrix.

\section{Signal Model}

Considering the coprime planar array [1], the model is made up of two URAs shown in Figure 1. Subarray 1 has $M_{1} \times M_{1}$ sensors and subarray 2 has $M_{2} \times M_{2}$ sensors, where $M_{1}$ and $M_{2}$ are coprime integers (assuming $M_{1}<M_{2}$ ). The $i$ th $(i=1,2)$ subarray sets the space distance between the two adjacent sensors as $M_{\bar{i}} \lambda / 2(\bar{i}=1,2$ and $\bar{i} \neq i)$, where $\lambda$ is the wavelength of impinging signals. The two subarrays coincide at the origin, so the total number of sensors is $M=M_{1}^{2}+M_{2}^{2}-1$. Suppose that there are $K$ narrowband far-field independent signals impinging on the array with the power $\left\{\sigma_{1}^{2}, \sigma_{2}^{2}, \ldots, \sigma_{K}^{2}\right\}$. The $k$ th signal comes from the elevation angle $\theta_{k}$ and azimuth angle $\varphi_{k}$. Hence, the received signals at the $i$ th subarray can be represented as

$$
\mathbf{X}_{i}(t)=\mathbf{A}_{i}(\varphi, \theta) \mathbf{S}(t)+\mathbf{N}_{i}(t) .
$$

The array manifold matrix is denoted as

$$
\mathbf{A}_{i}(\varphi, \theta)=\left[\begin{array}{lll}
\mathbf{a}_{i}\left(\varphi_{1}, \theta_{1}\right) & \cdots & \mathbf{a}_{i}\left(\varphi_{K}, \theta_{K}\right)
\end{array}\right],
$$

where $\mathbf{a}_{i}\left(\varphi_{k}, \theta_{k}\right)$ is a steering vector of size $M_{i}^{2} \times 1$ and the element in the $\left(x+M_{i} y+1\right)$ th $\left(0 \leq x, y \leq M_{i}-1\right)$ row is represented as $e^{j \pi M_{i} \sin \theta\left(x \cos \varphi_{k}+y \sin \varphi_{k}\right)}$. The signal data vector

$$
\mathbf{S}(t)=\left[s_{1}(t), \ldots, s_{K}(t)\right]^{T} .
$$

$t$ denotes the sampling time, where $t=1, \ldots, J$ and $J$ is the number of snapshots. And the noise vector

$$
\mathbf{N}_{i}(t)=\left[n_{1}(t), \ldots, n_{M_{i}^{2}}(t)\right]^{T},
$$


where $n_{m}(t)$ is usually Gaussian random variables with zero means and variance $\sigma_{n}^{2}$.

We then can calculate the covariance matrix of received signals given by

$$
\mathbf{R}_{\mathbf{X}_{i}}=\frac{1}{J} \mathbf{X}_{i} \mathbf{X}_{i}^{H}=\mathbf{A}_{i} \mathbf{R}_{\mathbf{S}} \mathbf{A}_{i}^{H}+\sigma_{n}^{2} \mathbf{I}_{M_{i}^{2}},
$$

where $\mathbf{R}_{\mathbf{S}}=\operatorname{diag}\left[\sigma_{1}^{2}, \ldots, \sigma_{K}^{2}\right]$. Apply $2 \mathrm{D}$ multiple signal classification (2D-MUSIC) $[8,26]$ to this covariance matrix and obtain the spatial spectrum function expressed as

$$
P_{i}(\varphi, \theta)=\left\|\mathbf{U}_{i, n}^{H} \mathbf{a}_{i}(\varphi, \theta)\right\|_{2}^{-2},
$$

where $\mathbf{U}_{i, n}$ is the noise subspace of the $i$ th subarray. Because the distance of adjacent sensors in each subarray is over half wavelength of impinging signals, through finding the peaks of spectrum, we can obtain the both ambiguous angles and real angles. But the common values of the estimated values of two subarrays are the real values.

\section{Fast DOA Estimation of Multiple Signals}

Unitary-ESPRIT is an efficient method, which solves the closed-form solutions of estimated values and reduces much complexity compared with the spectral peak search method, such as 2D-MUSIC. Unfortunately, Unitary-ESPRIT is commonly used in nonsparse arrays and it cannot estimate all ambiguous and real values.

3.1. Relationship between the Ambiguous Values and Real Values. Assume that there is a signal from the direction $\left(\varphi_{k}, \theta_{k}\right)$ and one estimated ambiguous value is $\left(\varphi_{i, k}^{a}, \theta_{i, k}^{a}\right)$. Hence, the relationship between the real angle and one ambiguous angle of the $i$ th subarray can be given by

$$
\begin{aligned}
& \pi M_{\bar{i}} \sin \theta_{k} \cos \varphi_{k}=2 \pi D_{x}+\pi M_{\bar{i}} \sin \theta_{i, k}^{a} \cos \varphi_{i, k}^{a} \\
& \pi M_{\bar{i}} \sin \theta_{k} \sin \varphi_{k}=2 \pi D_{y}+\pi M_{\bar{i}} \sin \theta_{i, k}^{a} \sin \varphi_{i, k}^{a} .
\end{aligned}
$$

Since $\varphi_{k} \in[-\pi, \pi]$ and $\theta_{k} \in[0, \pi / 2]$, we have $-1 \leq$ $\sin \theta_{k} \cos \varphi_{k} \leq 1$ and $-1 \leq \sin \theta_{k} \sin \varphi_{k} \leq 1$. Thus, we have

$$
\begin{aligned}
& 2 D_{x}=M_{\bar{i}} \sin \theta_{k} \cos \varphi_{k}-M_{\bar{i}} \sin \theta_{k}^{a} \cos \varphi_{k}^{a} \\
& 2 D_{y}=M_{\bar{i}} \sin \theta_{k} \sin \varphi_{k}-M_{\bar{i}} \sin \theta_{k}^{a} \sin \varphi_{k}^{a},
\end{aligned}
$$

and $D_{x}$ and $D_{y}$ are the integers in the ranges of $\left[-M_{\bar{i}}, M_{\bar{i}}\right]$. As a result, when we can obtain one estimation angle $\left(\widehat{\varphi}_{i, k}, \widehat{\theta}_{i, k}\right)$, no matter the real angle or one ambiguous angle, we can obtain

$$
\begin{aligned}
\Theta_{i, k} & =\left\{\mu_{i, k, \ell} \mid \mu_{i, k, \ell}=\frac{2 D_{x, \ell}}{M_{\bar{i}}}+\sin \widehat{\theta}_{i, k} \cos \widehat{\varphi}_{i, k}, D_{x, \ell}\right. \\
\in & {\left.\left[-M_{\bar{i}}, M_{\bar{i}}\right]\right\} } \\
\Phi_{i, k} & =\left\{v_{i, k, \ell} \mid v_{i, k, \ell}=\frac{2 D_{y, \ell}}{M_{\bar{i}}}+\sin \widehat{\theta}_{i, k} \cos \widehat{\varphi}_{i, k}, D_{y, \ell}\right. \\
\in & {\left.\left[-M_{\bar{i}}, M_{\bar{i}}\right]\right\}, }
\end{aligned}
$$

where $\Theta_{i, k}$ and $\Phi_{i, k}$ are the sets with $2 M_{\bar{i}}+1$ values containing the unique real value. Combine all the sets and define

$$
\begin{aligned}
& \Theta_{i}=\left\{\Theta_{i, 1}, \ldots, \Theta_{i, K}\right\} \\
& \Phi_{i}=\left\{\Phi_{i, 1}, \ldots, \Phi_{i, K}\right\} .
\end{aligned}
$$

Through finding common values between $\Theta_{2, k}$ and $\Theta_{1}$, $\Phi_{2, k}$ and $\Phi_{1}(k=1, \ldots, K)$, we can finally select the corresponding true value as $\left(\mu_{k}^{r}, v_{k}^{r}\right)$.

3.2. Unitary-ESPRIT for DOA Estimation. We can use Unitary-ESPRIT to obtain one possible estimated value of each signal. First, we define $\Pi_{q}$ as a inverse matrix of size $q \times q$, whose counter-diagonal values are 1 while the others are 0 . Thus, the unitary matrix $\mathbf{Q}$ satisfies $\Pi_{q} \mathbf{Q}^{*}=\mathbf{Q}$. For example, the unitary matrix of odd order is expressed as

$$
\mathbf{Q}_{2 q+1}=\frac{1}{\sqrt{2}}\left[\begin{array}{ccc}
\mathbf{I}_{q} & \mathbf{O} & j \mathbf{I}_{q} \\
\mathbf{O}^{T} & \sqrt{2} & \mathbf{O}^{T} \\
\boldsymbol{\Pi}_{q} & \mathbf{O} & -j \boldsymbol{\Pi}_{q}
\end{array}\right] .
$$

Moreover, the matrix of even order is obtained from (11) by dropping its center row and center column. We then transform the complex-value covariance matrix to real-value covariance matrix as

$$
\mathbf{R}_{\mathbf{Z}_{i}}=\mathbf{Q}_{M_{i}^{2}}^{H} \mathbf{R}_{\mathbf{X}_{i}} \mathbf{Q}_{M_{i}^{2}} \cdot
$$

Next, we define the selection matrices $\mathbf{F}_{1 i}=\left[\begin{array}{ll}\mathbf{I}_{M_{i}-1} & \mathbf{O}\end{array}\right]$ and $\mathbf{F}_{2 i}=\left[\begin{array}{ll}\mathbf{O} & \mathbf{I}_{M_{i}-1}\end{array}\right]$ and construct the desired $M_{i}\left(M_{i}-1\right) \times M_{i}^{2}$ dimensional selection matrices as $\mathbf{J}_{1 i}=\mathbf{I}_{M_{i}} \otimes \mathbf{F}_{1 i}$ and $\mathbf{J}_{2 i}=\mathbf{F}_{2 i} \otimes$ $\mathbf{I}_{M_{i}}$, where $\otimes$ represents the Kronecker product. Therefore, we define

$$
\begin{aligned}
& \mathbf{K}_{\mu 1 i}=2 \operatorname{Re}\left(\mathbf{Q}_{M_{i}\left(M_{i}-1\right)}^{H} \mathbf{J}_{1 i} \mathbf{Q}_{M_{i}^{2}}\right), \\
& \mathbf{K}_{\mu 2 i}=2 \operatorname{Im}\left(\mathbf{Q}_{M_{i}\left(M_{i}-1\right)}^{H} \mathbf{J}_{1 i} \mathbf{Q}_{M_{i}^{2}}\right), \\
& \mathbf{K}_{\nu 1 i}=2 \operatorname{Re}\left(\mathbf{Q}_{M_{i}\left(M_{i}-1\right)}^{H} \mathbf{J}_{2 i} \mathbf{Q}_{M_{i}^{2}}\right), \\
& \mathbf{K}_{\mu 2 i}=2 \operatorname{Im}\left(\mathbf{Q}_{M_{i}\left(M_{i}-1\right)}^{H} \mathbf{J}_{2 i} \mathbf{Q}_{M_{i}^{2}}\right),
\end{aligned}
$$

where $\operatorname{Re}[\bullet]$ and $\operatorname{Im}[\bullet]$ represent the real part and imaginary part of the matrix or vector, respectively. Take eigenvalue 
decomposition of $\mathbf{R}_{\mathbf{Z}_{i}}$ and obtain the signal subspace $\mathbf{U}_{S_{i}}$. At last, we combine the last two steps and have

$$
\begin{gathered}
\boldsymbol{\Psi}_{\mu i}=\left(\mathbf{K}_{\mu 1 i} \mathbf{U}_{S_{i}}\right)^{+} \mathbf{K}_{\mu 2 i} \mathbf{U}_{S_{i}}, \\
\boldsymbol{\Psi}_{v i}=\left(\mathbf{K}_{v 1 i} \mathbf{U}_{S_{i}}\right)^{+} \mathbf{K}_{v 2 i} \mathbf{U}_{S_{i}},
\end{gathered}
$$

where $(\bullet)^{+}$is denoted as pseudoinverse operator. Define $\Psi_{i}=$ $\Psi_{\mu i}+j \Psi_{v i}$, and calculate its eigenvalue vector $\lambda_{i}$. We can acquire the values $\widehat{\mu}_{i, k}=2 \arctan \left(\operatorname{Re}\left(\lambda_{i, k}\right)\right) / \pi$ and $\hat{v}_{i, k}=$ $2 \arctan \left(\operatorname{Im}\left(\lambda_{i, k}\right)\right) / \pi$, which are denoted as

$$
\begin{aligned}
\hat{\mu}_{i, k} & =\sin \widehat{\theta}_{i, k} \cos \widehat{\varphi}_{i, k} \\
\widehat{v}_{i, k} & =\sin \widehat{\theta}_{i, k} \sin \widehat{\varphi}_{i, k} .
\end{aligned}
$$

Hence, considering (9) and (10), we can remove all ambiguous values and obtain the corresponding true values $\left(\widehat{\mu}_{k}^{r}, \widehat{v}_{k}^{r}\right)$. The final estimated DOAs are defined as

$$
\begin{aligned}
& \widehat{\theta}_{k}^{r}=\arcsin \left(\sqrt{\left(\widehat{\mu}_{k}^{r}\right)^{2}+\left(\widehat{\nu}_{k}^{r}\right)^{2}}\right) \\
& \hat{\varphi}_{k}^{r}=\arctan \left(\frac{\widehat{v}_{k}^{r}}{\widehat{\mu}_{k}^{r}}\right) .
\end{aligned}
$$

3.3. DOA Estimation for Noncircular Signals. The last subsection presents the algorithm for circular signals, which can also be used for general independent signals. When there are noncircular signals, the ellipse covariance matrix of them can be nonzero; thus, we need add some additional procession. We assume that the signals are strictly noncircular. At first, we can "double" the available sensors by

$$
\mathbf{X}_{i}^{(n c)}=\left[\begin{array}{c}
\mathbf{X}_{i} \\
\boldsymbol{\Pi}_{M_{i}^{2}} \mathbf{X}_{i}^{*}
\end{array}\right] .
$$

Let us define

$$
\mathbf{Z}_{i}^{(n c)}=\left[\begin{array}{ll}
\mathbf{X}_{i}^{(n c)} & \Pi_{2 M_{i}^{2}} \mathbf{X}_{i}^{(n c) *} \Pi_{J}
\end{array}\right]
$$

where $\mathbf{Q}_{2 M_{i}^{2}}^{H} \mathbf{Z}_{i}^{(n c)} \mathbf{Q}_{2 J}$ can be a real-value matrix. The covariance matrix can be given by

$$
\mathbf{R}_{\mathbf{Z}_{i}}^{(n c)}=\frac{1}{2 J} \mathbf{Q}_{2 M_{i}^{2}}^{H} \mathbf{Z}_{i}^{(n c)} \mathbf{Z}_{i}^{(n c) H} \mathbf{Q}_{2 M_{i}^{2}} .
$$

Next, the selection matrices should be modified in the following fashion:

$$
\mathbf{J}_{p i}^{(n c)}=\left[\begin{array}{ll}
1 & 0 \\
0 & 1
\end{array}\right] \otimes \mathbf{J}_{p i}, \quad(p=1,2) .
$$

We then have

$$
\begin{aligned}
& \mathbf{K}_{\mu 1 i}^{(n c)}=2 \operatorname{Re}\left(\mathbf{Q}_{2 M_{i}\left(M_{i}-1\right)}^{H} \mathbf{J}_{1 i}^{(n c)} \mathbf{Q}_{2 M_{i}^{2}}\right), \\
& \mathbf{K}_{\mu 2 i}^{(n c)}=2 \operatorname{Im}\left(\mathbf{Q}_{2 M_{i}\left(M_{i}-1\right)}^{H} \mathbf{J}_{1 i}^{(n c)} \mathbf{Q}_{2 M_{i}^{2}}\right), \\
& \mathbf{K}_{\nu 1 i}^{(n c)}=2 \operatorname{Re}\left(\mathbf{Q}_{2 M_{i}\left(M_{i}-1\right)} \mathbf{J}_{2 i}^{(n c)} \mathbf{Q}_{2 M_{i}^{2}}\right), \\
& \mathbf{K}_{v 2 i}^{(n c)}=2 \operatorname{Im}\left(\mathbf{Q}_{2 M_{i}\left(M_{i}-1\right)}^{H} \mathbf{J}_{2 i}^{(n c)} \mathbf{Q}_{2 M_{i}^{2}}\right) .
\end{aligned}
$$

Take eigenvalue decomposition of $\mathbf{R}_{\mathbf{Z}_{i}}^{(n c)}$ and obtain the signal subspace $\mathbf{U}_{S_{i}}^{(n c)}$. We combine the last two steps and finally have

$$
\begin{gathered}
\Psi_{\mu i}^{(n c)}=\left(\mathbf{K}_{\mu 1 i}^{(n c)} \mathbf{U}_{S_{i}}^{(n c)}\right)^{+} \mathbf{K}_{\mu 2 i}^{(n c)} \mathbf{U}_{S_{i}}^{(n c)}, \\
\Psi_{v i}^{(n c)}=\left(\mathbf{K}_{v 1 i}^{(n c)} \mathbf{U}_{S_{i}}^{(n c)}\right)^{+} \mathbf{K}_{v 2 i}^{(n c)} \mathbf{U}_{S_{i}}^{(n c)},
\end{gathered}
$$

Define $\Psi_{i}^{(n c)}=\Psi_{\mu i}^{(n c)}+j \Psi_{v i}^{(n c)}$, and calculate its eigenvalue vector $\lambda_{i}^{(n c)}$. We can acquire the values $\mu_{i, k}^{(n c)}=$ $2 \arctan \left(\operatorname{Re}\left(\lambda_{i, k}^{(n c)}\right)\right) / \pi$ and $\gamma_{i, k}^{(n c)}=2 \arctan \left(\operatorname{Im}\left(\lambda_{i, k}^{(n c)}\right)\right) / \pi$. Similarly, considering (9) and (10), we can obtain the corresponding true values $\left(\widehat{\mu}_{k}^{r,(n c)}, \hat{v}_{k}^{r,(n c)}\right)$. And the estimation $\left(\widehat{\theta}_{k}^{r}, \widehat{\varphi}_{k}^{r}\right)$ can be calculated via (20).

3.4. Algorithm Steps Conclusion. A detailed flowchart of this algorithm is shown in Figure 2. The main steps of the proposed algorithm can be summarized as follows:

Step 1. Calculate the covariance matrix via (5).

Step 2. Apply Unitary-ESPRIT and obtain the estimation $\left(\widehat{\mu}_{i, k}, \widehat{v}_{i, k}\right)(k=1, \ldots, K)$.

Step 3. If the signals are noncircular, we transform the received signal data as (22) and then apply NC-UnitaryESPRIT to obtain the estimation $\left(\hat{\mu}_{i, k}^{(n c)}, \widehat{v}_{i, k}^{(n c)}\right)(k=1, \ldots, K)$.

Step 4. Calculate all real values and ambiguous values through (9) and (10) and find the corresponding real values as $\left(\widehat{\mu}_{k}^{r}, \widehat{v}_{k}^{r}\right)$ or $\left(\widehat{\mu}_{k}^{r,(n c)}, \widehat{v}_{k}^{r,(n c)}\right)$.

Step 5. Resolve the DOA estimation $\left(\widehat{\theta}_{k}, \widehat{\varphi}_{k}\right)$ via (20).

\section{Analysis of Computational Complexity and DOF}

4.1. Analysis of Computational Complexity. We first analyze the computational complexity of the proposed method using Unitary-ESPRIT algorithm and compare it with the spectral peak search method based on 2D-MUSIC algorithm and PSS. And then analyze the complexity of proposed method using NC-Unitary-ESPRIT when signals are noncircular.

The complexity of the proposed method using UnitaryESPRIT mainly concludes three parts. The complexities of calculating the covariance matrix, eigenvalue decomposition, and Unitary-ESPRIT are $O\left(4 J\left(M_{1}^{4}+M_{2}^{4}\right)\right), O\left(\left(M_{1}^{2}\right)^{3}+\left(M_{2}^{2}\right)^{3}\right)$, and $O\left(\left(2 M_{1}^{2}\right)^{3}+\left(2 M_{2}^{2}\right)^{3}+2 K^{3}\right)$, respectively. Thus, the total complexity of proposed algorithm using Unitary-ESPRIT is $O\left(4 J\left(M_{1}^{4}+M_{2}^{4}\right)+9\left(M_{1}^{6}+M_{2}^{6}\right)+2 K^{3}\right)$. And 2D-MUSIC and PSS cost the complexities as $O\left(4 J\left(M_{1}^{4}+M_{2}^{4}\right)+M_{1}^{6}+\right.$ $\left.M_{2}^{6}+G_{\varphi} G_{\theta}\left(M_{1}^{4}+M_{2}^{4}\right)\right)$ and $O\left(4 J\left(M_{1}^{4}+M_{2}^{4}\right)+M_{1}^{6}+M_{2}^{6}+\right.$ $\left.G_{\varphi} G_{\theta}\left(M_{1}^{4} / M_{2}^{2}+M_{2}^{4} / M_{1}^{2}\right)\right)$, respectively, where $G_{\varphi}$ and $G_{\theta}$ denote the spectral points. Similarly, the complexity of the proposed method using NC-Unitary-ESPRIT also has three parts: calculating the covariance matrix as $O\left(16 J\left(M_{1}^{4}+M_{2}^{4}\right)\right)$ ), 


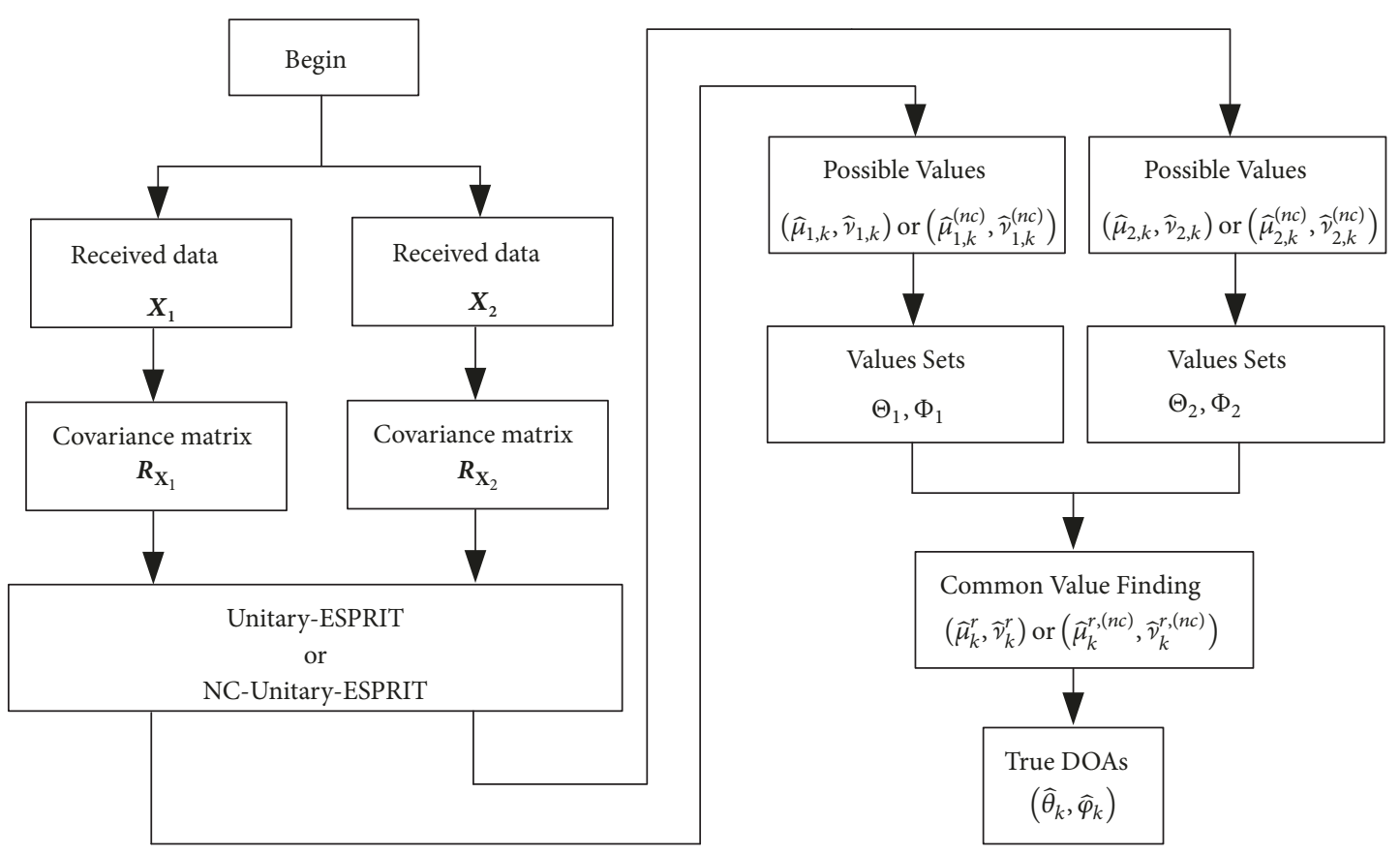

FIGURE 2: Flowchart of the proposed algorithm.

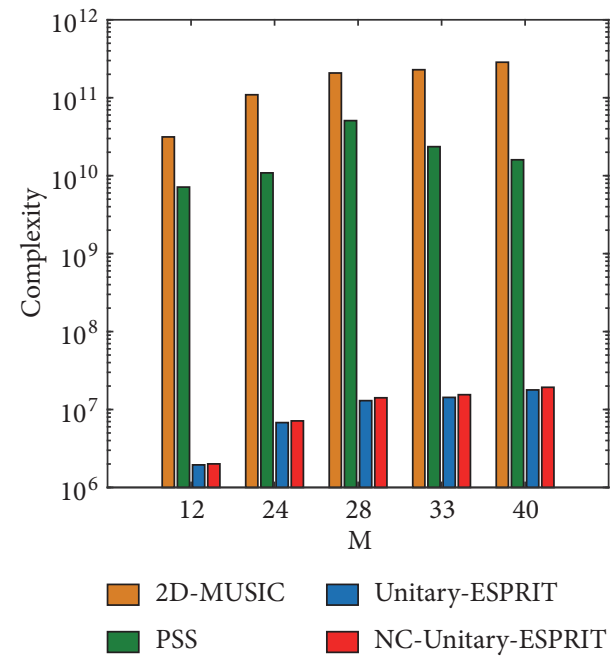

(a)

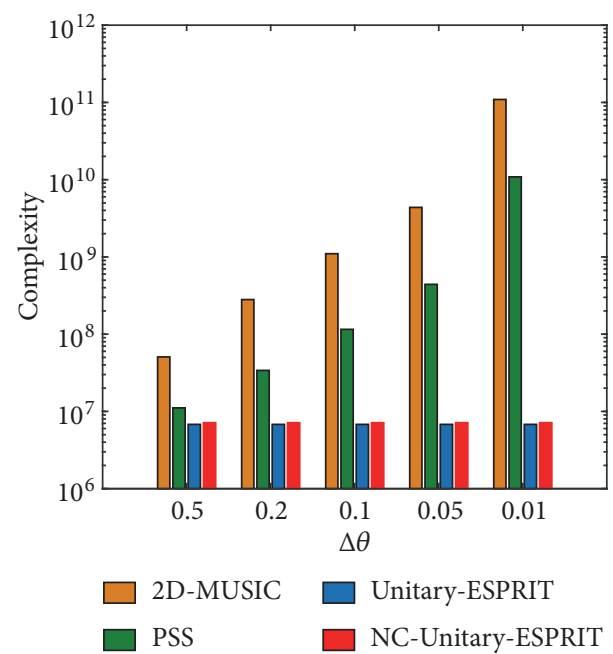

(b)

Figure 3: Complexity comparison: (a) versus the number of sensors when $J=5000$ and $\Delta \theta=0.01^{\circ}$; (b) versus $\Delta \theta$ when $M_{1}=3, M_{2}=4$, and $J=5000$.

eigenvalue decomposition as $O\left(\left(2 M_{1}^{2}\right)^{3}+\left(2 M_{2}^{2}\right)^{3}\right)$, and NCUnitary-ESPRIT as $O\left(\left(4 M_{1}^{2}\right)^{3}+\left(4 M_{2}^{2}\right)^{3}+4 K^{3}\right)$. Hence, the total complexity of proposed algorithm using NC-UnitaryESPRIT is $O\left(16 J\left(M_{1}^{4}+M_{2}^{4}\right)+72\left(M_{1}^{6}+M_{2}^{6}\right)+4 K^{3}\right)$. For the sake of clarity, the computational complexities of all these approaches are summarized in Table 1. Moreover, we compare the complexities of methods versus the number of sensors $(M)$ and the searching step $\left(\Delta \theta=\Delta \varphi\right.$, where $G_{\theta}=$ $\left.90 / \Delta \theta+1, G_{\varphi}=360 / \Delta \varphi+1\right)$ in Figures 3(a) and 3(b), respectively.
As shown in Figure 3, compared with the spectral peak search methods, the proposed algorithms using UnitaryESPRIT and NC-Unitary-ESPRIT have reduced much complexity. The searching step has an impact on the spectral peak search methods but no influence to proposed algorithms. Hence, the proposed algorithms can obtain the high accuracy while the spectral peak search methods need a small searching step and cost more complexity to realize that. Moreover, because the method for noncircular signals expands the sensors, the complexity of NC-Unitary-ESPRIT 
TABLE 1: Computational complexity comparison.

\begin{tabular}{lc}
\hline Algorithm & Complexity \\
\hline 2D-MUSIC & $O\left(4 J\left(M_{1}^{4}+M_{2}^{4}\right)+M_{1}^{6}+M_{2}^{6}+G_{\varphi} G_{\theta}\left(M_{1}^{4}+M_{2}^{4}\right)\right)$ \\
PSS & $O\left(4 J\left(M_{1}^{4}+M_{2}^{4}\right)+M_{1}^{6}+M_{2}^{6}+G_{\varphi} G_{\theta}\left(\frac{M_{1}^{4}}{M_{2}^{2}}+\frac{M_{2}^{4}}{M_{1}^{2}}\right)\right)$ \\
Unitary-ESPRIT & $O\left(4 J\left(M_{1}^{4}+M_{2}^{4}\right)+9\left(M_{1}^{6}+M_{2}^{6}\right)+2 K^{3}\right)$ \\
NC-Unitary-ESPRIT & $O\left(16 J\left(M_{1}^{4}+M_{2}^{4}\right)+72\left(M_{1}^{6}+M_{2}^{6}\right)+4 K^{3}\right)$ \\
\hline
\end{tabular}

TABLE 2: The detailed values of each step.

\begin{tabular}{lcc}
\hline Value & Subarray 1 & Subarray 2 \\
\hline$\widehat{\mu}_{i}$ & -0.1465 & -0.3131 \\
$\widehat{v}_{i}$ & -0.1465 & -0.3131 \\
$\Theta_{i}$ & $-2.1465,-1.6465,-1.1465,-0.6465,-0.1465,0.3535,0.8535,1.3535,1.8535$ & $-2.3131,-1.6464,-0.9798,-0.3131,0.3536,1.0202,1.6869$ \\
$\Phi_{i}$ & $-2.1465,-1.6465,-1.1465,-0.6465,-0.1465,0.3535,0.8535,1.3535,1.8535$ & $-2.3131,-1.6464,-0.9798,-0.3131,0.3536,1.0202,1.6869$ \\
\hline
\end{tabular}

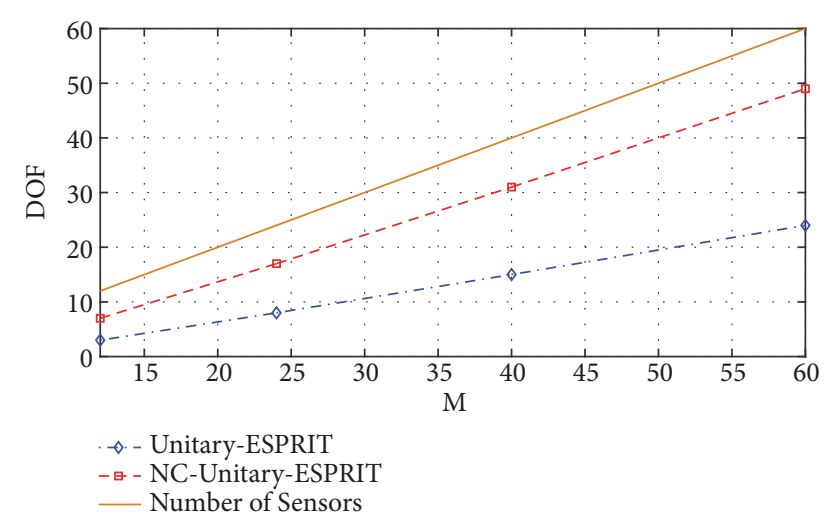

Figure 4: DOF comparison versus the number of sensors.

is higher than Unitary-ESPRIT, but the gap is not big. We can obtain a higher precise estimation of noncircular signals with a little complexity increase.

4.2. Analysis of DOF. The DOF determines the maximum number of signals that we can estimate directly. 2D-MUSIC, PSS and the proposed algorithms are all common values finding method to resolve the DOAs, where the DOFs of those algorithms are limited by the number of subarray sensors. The DOFs of 2D-MUSIC, PSS, and proposed algorithm using Unitary-ESPRIT are equal as $M_{1}^{2}-1$. When the signals are noncircular, because NC-Unitary-ESPRIT can "double" the sensors, the DOF of this method is $2 M_{1}^{2}-1$. The detailed values are presented in Figure 4. The figure shows that NCUnitary-ESPRIT does increase the DOF compared with the Unitary-ESPRIT. But DOFs of the proposed algorithms are both smaller than the number of sensors, which means that DOF may be further improved with one proper method.

\section{Simulation Results}

This section performs the results of simulation experiments comparing the two proposed methods with PSS. To measure the accuracy of the algorithms, define the root mean square error (RMSE) as

$$
\mathrm{RMSE}=\sqrt{\frac{1}{L K} \sum_{\ell=1}^{L}\left\|\boldsymbol{\xi}-\widehat{\boldsymbol{\xi}}_{\ell}\right\|^{2}}
$$

where $L, \boldsymbol{\xi}$, and $\widehat{\boldsymbol{\xi}}_{\boldsymbol{i}}$ are the number of Monte Carlo simulations, the $\ell$ th real values and the estimated values, respectively. We assume that the impinging noncircular signals are BPSK modulation. And if the impinging signals are circular signals, they are QPSK modulation.

(1) Feasibility Demonstration of Proposed Algorithms. We assume that there is one impinging signal from $(\theta, \varphi)=$ $\left(30^{\circ}, 45^{\circ}\right)$. We use Unitary-ESPRIT to resolve the DOA and the results of each step are listed in Table 2. Obviously, $\left(\widehat{\mu}_{k}^{r}, \widehat{v}_{k}^{r}\right)=(0.3536,0.3536)$, and then $\left(\widehat{\theta}_{k}^{r}, \widehat{\varphi}_{k}^{r}\right)=$ $\left(30.0029^{\circ}, 45.0001^{\circ}\right)$. Hence, we prove that the proposed algorithm can estimate DOA without the spectral peak search.

(2) Performance of Estimating Multiple Signals. The two proposed methods can estimate multiple signals. To verify this ability, we set $M_{1}=3$ and $M_{2}=4(M=24)$ and consider the case of $K=7$ circular signals and $K=16$ noncircular signals with a signal-of-noise ratio (SNR) of $15 \mathrm{~dB}$ and $J=5000$, respectively. The distribution of estimated values from 100 simulations using two proposed methods is presented in Figures 5(a) and 5(b), respectively. The figure demonstrates that the proposed algorithms can completely estimate DOAs of multiple signals. Moreover, the estimated values are all concentrated around the real values.

(3) RMSE Comparison under Different SNRs. In this simulation, we study the RMSE performance of proposed algorithms under different SNRs in the setting with $L=100$, $J=5000$ and SNRs from $-5 \mathrm{~dB}$ to $15 \mathrm{~dB}$ at $5 \mathrm{~dB}$ intervals, compared with PSS. We set that there are $K=6$ signals which are strictly noncircular, where both Unitary-ESPRIT and NCUnitary-ESPRIT can be applied. The results are shown in Figure 6. We can see that the RMSE of Unitary-ESPRIT is 


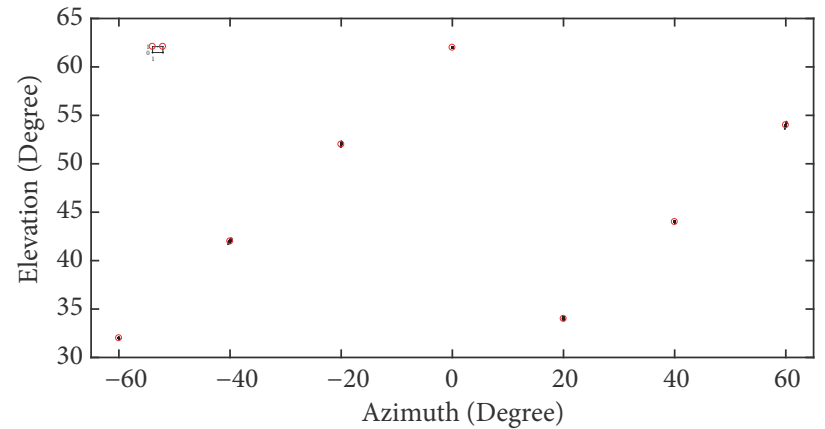

Estimated Value - Real Value

(a) $K=7$ circular signals

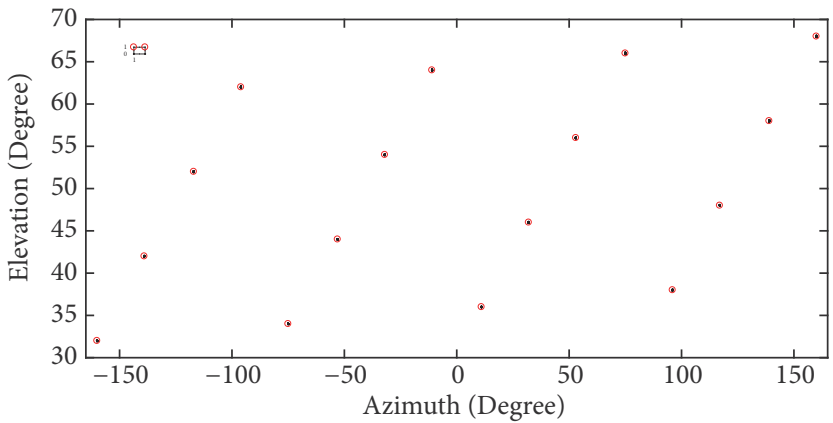

Estimated Value - Real Value

(b) $K=16$ noncircular signals

FIGURE 5: Distribution of azimuth and elevation estimation (a) Unitary-ESPRIT (b) NC-Unitary-ESPRIT.

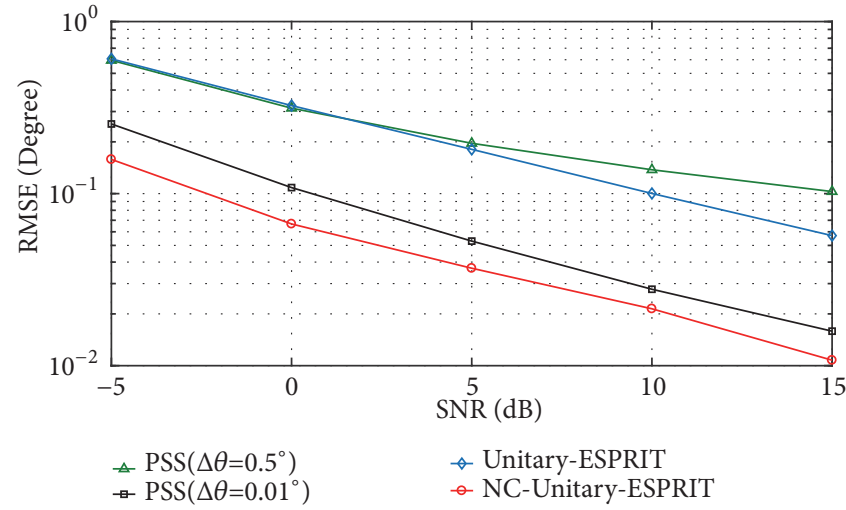

(a)

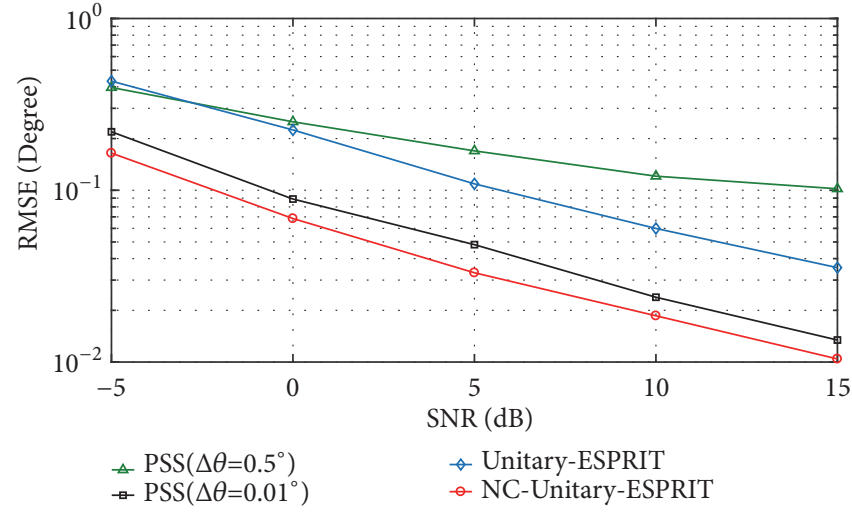

(b)

FIGURE 6: RMSE comparison under different SNRs (a) elevation (b) azimuth.

smaller than that of PSS with the searching step $\Delta \theta=0.5^{\circ}$, and the gap between them become bigger with the increase of SNR. However, when the searching step of PSS is $\Delta \theta=$ $0.01^{\circ}$, the RMSE of it is much smaller. But considering the complexity, that of PSS is increased from $O\left(1.11 \times 10^{7}\right)$ to $O\left(1.08 \times 10^{10}\right)$ while that of Unitary-ESPRIT is only $O(6.79 \times$ $10^{6}$ ), which means that PSS needs to cost much complexity to improve the accuracy. When the signals are noncircular, we apply the NC-Unitary-ESPRIT with the cost of complexity as $O\left(7.14 \times 10^{6}\right)$ and obtain the highest accuracy, which is computationally efficient and favorable.

In addition, another advantage of NC-Unitary-ESPRIT is that it can estimate more signals than PSS and UnitaryESPRIT. Hence, we set four situations where the number of noncircular signals is $K=6, K=9, K=12$, and $K=$ 16 , respectively. The RMSEs of those different situations are presented in Figure 7. When the number of signals is bigger than that of subarray sensors, it can still maintain the high accuracy.

(4) RMSE Comparison under Different Number of Snapshots. Consider situations used in simulation (3) again. The number of snapshots is varied in the range from $J=$
$[50,100,200,500,1000,2000,5000,10000]$, when SNR = $15 \mathrm{~dB}$, and the results are shown in Figure 8. With the increase of number of snapshots, the accuracy of the proposed algorithms can be higher. Moreover, the decline gradually reaches a plateau. The other conclusions are the same as those from simulation (3).

\section{Conclusions}

The paper has proposed a fast DOA estimation approach in a coprime planar array for multiple signals, where circular and noncircular signals are concerned. The paper has described the model and the associated algorithms and analyzed the computational complexity as well as DOF of the proposed methods in comparison with that of existing algorithms. Through the theoretical analysis and simulation experiments, we demonstrate that proposed methods can detect multiple signals and realize the estimation of DOAs. Unitary-ESPRIT can obtain a close accuracy as the spectral peak search methods. Although the spectral peak search methods can obtain a higher accuracy with a small searching step, it costs much larger complexity than Unitary-ESPRIT. Moreover, when we estimate the DOAs of strictly noncircular signals, 


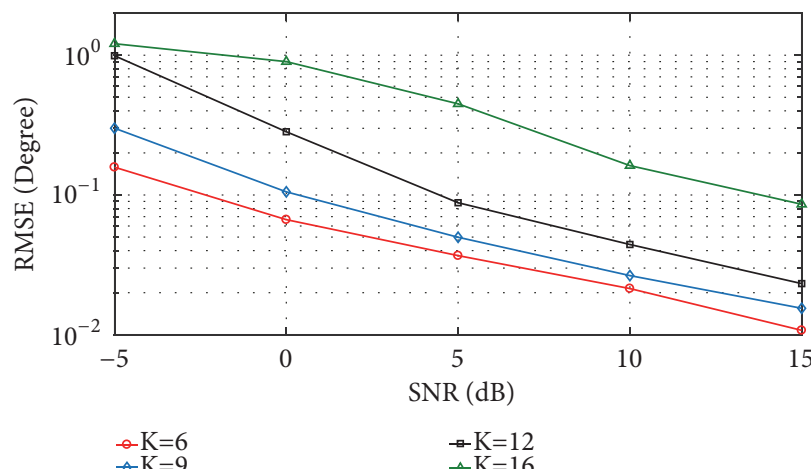

(a)

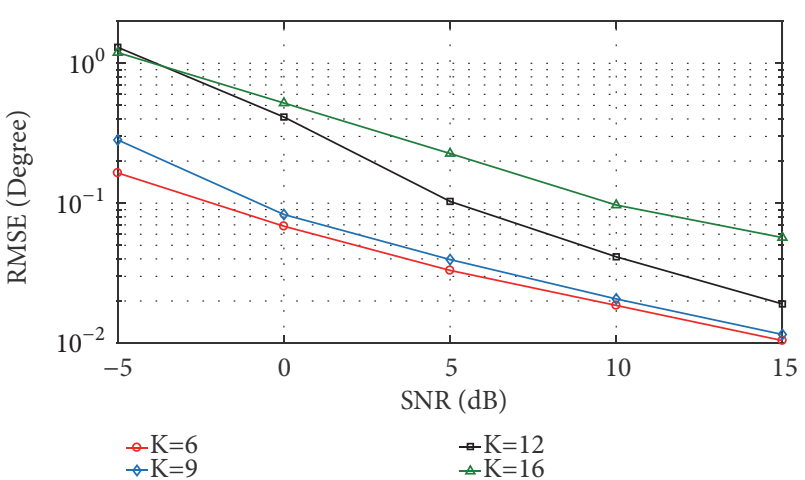

(b)

FIGURE 7: RMSE of NC-Unitary-ESPRIT under different number of noncircular signals (a) elevation (b) azimuth.

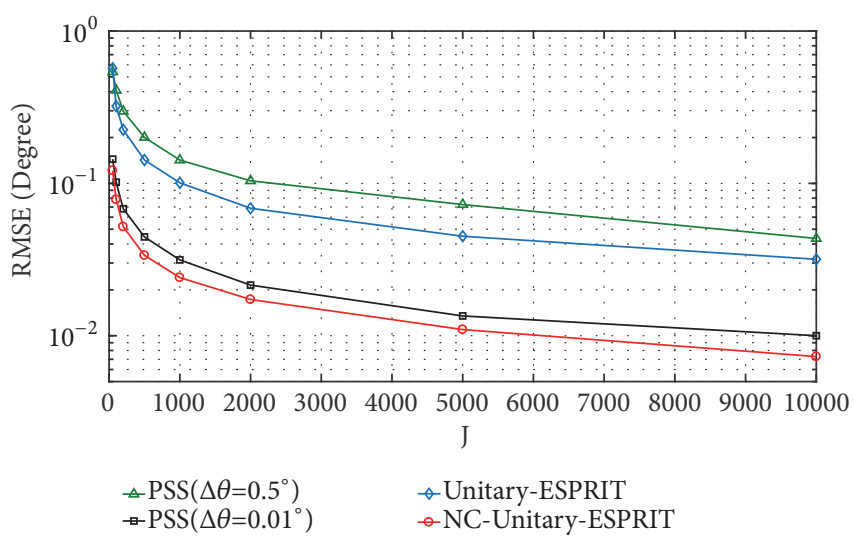

(a)

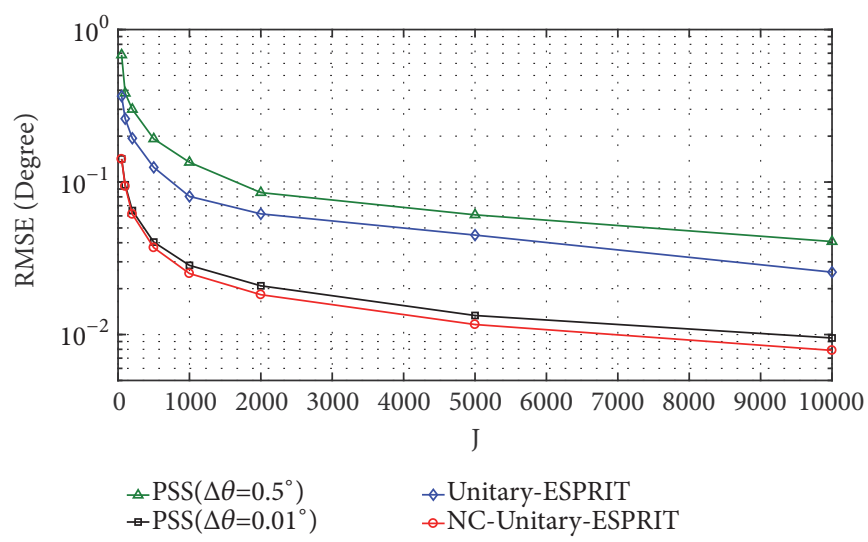

(b)

FIGURE 8: RMSE comparison under different number of snapshots (a) elevation (b) azimuth.

NC-Unitary-ESPRIT is favorable in both accuracy and DOF compared with existing algorithms and Unitary-ESPRIT. In practice, there can be the coexistence of both circular and noncircular signals. Hence, how to separate the two kinds of signals and resolve their DOAs with the consideration of signals features deserves the further research.

\section{Data Availability}

The data, which are produced by simulations, used to support the findings of this study are available from the corresponding author upon request.

\section{Conflicts of Interest}

The authors declare that they have no conflicts of interest.

\section{Acknowledgments}

The work is supported by the National Natural Science Foundation of China (Grant no. 61401513).

\section{References}

[1] H. Xu, Y. Zhang, B. Ba, D. Wang, and X. Li, “Two-dimensional direction-of-arrival fast estimation of multiple signals with matrix completion theory in coprime planar array," Sensors, vol. 18, no. 6, p. 1741, 2018.

[2] J. Ball and N. Younan, "Radar and radio signal processing," Electronics, vol. 6, no. 3, p. 64, 2017.

[3] Y. L. Wang, S. L. Ma, G. L. Liang, and Z. Fan, "Chirp spread spectrum of orthogonal frequency division multiplexing underwater acoustic communication system based on multipath diversity receive," Acta Physica Sinica, vol. 63, no. 4, Article ID 044302, 2014.

[4] Y. Zhou, Y. Y. Wu, D. S. Chen, and F. Tong, "Compressed sensing estimation of underwater acoustic mimo channels based on temporal joint sparse recovery," Journal of Electronics \& Information Technology, vol. 38, no. 3, pp. 1920-1927, 2016.

[5] H. S. Maghdid, I. A. Lami, K. Z. Ghafoor, and J. Lloret, "Seamless outdoors-indoors localization solutions on smartphones," ACM Computing Surveys, vol. 38, no. 3, pp. 1-34, 2016.

[6] M. Garcia, F. Boronat, J. Tomás, and J. Lloret, "The development of two systems for indoor wireless sensors self-location," Ad Hoc \& Sensor Wireless Networks, vol. 8, no. 3-4, pp. 235-258, 2009.

[7] A. Gaber and A. Omar, "Sub-nanosecond accuracy of TDOA estimation using Matrix Pencil algorithms and IEEE 802.11," 
in Proceedings of the 9th International Symposium on Wireless Communication Systems, ISWCS '12, pp. 646-650, France, August 2012.

[8] P. Heidenreich, A. M. Zoubir, and M. Rübsamen, "Joint 2-D DOA estimation and phase calibration for uniform rectangular arrays," IEEE Transactions on Signal Processing, vol. 60, no. 9, pp. 4683-4693, 2012.

[9] P. Pal and P. P. Vaidyanathan, "Coprime sampling and the music algorithm," in Proceedings of the IEEE Digital Signal Processing Workshop and IEEE Signal Processing Education Workshop (DSP/SPE '11), pp. 289-294, IEEE, Sedona, Ariz, USA, January 2011.

[10] P. P. Vaidyanathan and P. Pal, "Sparse sensing with co-prime samplers and arrays," IEEE Transactions on Signal Processing, vol. 59, no. 2, pp. 573-586, 2011.

[11] D. Zhang, Y. Zhang, G. Zheng, C. Feng, and J. Tang, "Improved DOA estimation algorithm for co-prime linear arrays using root-MUSIC algorithm," IEEE Electronics Letters, vol. 53, no. 18, pp. 1277-1279, 2017.

[12] C. Zhou and J. Zhou, "Direction-of-arrival estimation with coarray ESPRIT for coprime array," Sensors, vol. 17, no. 8, 2017.

[13] C. Zhou, Z. Shi, Y. Gu, and X. Shen, "DECOM: DOA estimation with combined MUSIC for coprime array," in Proceedings of the International Conference on Wireless Communications and Signal Processing, WCSP '13, pp. 1-5, China, 2013.

[14] Q. Wu, F. Sun, P. Lan, G. Ding, and X. Zhang, "Two-dimensional direction-of-arrival estimation for co-prime planar arrays: a partial spectral search approach," IEEE Sensors Journal, vol. 16, no. 14, pp. 5660-5670, 2016.

[15] H. Xu, D. Wang, B. Ba, W. Cui, and Y. Zhang, "Direction-ofarrival estimation for both uncorrelated and coherent signals in coprime array," IEEE Access, vol. 7, pp. 18590-18600, 2019.

[16] R. Ashri, H. Shaban, and M. A. El-Nasr, "A novel fractional fourier transform-based ASK-OFDM system for underwater acoustic communications," Applied Sciences (Switzerland), vol. 7, no. 12, p. 1286, 2017.

[17] C. T. Yen, J. F. Huang, and W. Z. Zhang, "Hiding stealth optical CDMA signals in public BPSK channels for optical wireless communication," Applied Sciences, vol. 8, no. 10, p. 1731, 2018.

[18] Y. Aikawa, "Optical comparison operation for 8-Bit QPSKmodulated signal by using serially-cascaded delay line interferometer," Applied Sciences, vol. 8, no. 9, p. 1440, 2018.

[19] W. Cui, Z. Dai, B. Ba, and H. Lu, "Fast DOA estimation of distributed noncircular sources by cross-correlation sampling decomposition," Dianzi Yu Xinxi Xuebao/Journal of Electronics and Information Technology, vol. 40, no. 5, pp. 1226-1233, 2018.

[20] R. Li, X. Shi, L. Chen et al., "The non-circular MUSIC method for uniform rectangular arrays," in Proceedings of the International Conference on Microwave and Millimeter Wave Technology, ICMMT '10, pp. 1390-1393, 2010.

[21] H. Y. Xu, D. M. Wang, S. Z. Lin, B. Ba, and Y. K. Zhang, “Twodimensional direction-of-arrival estimation of noncircular signals in coprime planar array with high degree of freedom," Mathematical Problems in Engineering, vol. 2019, Article ID 3078376, 10 pages, 2019.

[22] M. D. Zoltowski, M. Haardt, and C. P. Mathews, "Closed-form 2-D angle estimation with rectangular arrays in element space or beamspace via unitary ESPRIT," IEEE Transactions on Signal Processing, vol. 44, no. 2, pp. 316-328, 1996.

[23] M. Haardt and J. A. Nossek, "Unitary ESPRIT: how to obtain increased estimation accuracy with a reduced computational burden," IEEE Transactions on Signal Processing, vol. 43, no. 5, pp. 1232-1242, 1995.

[24] M. Haardt and F. Römer, "Enhancements of unitary ESPRIT for non-circular sources," in Proceedings of the IEEE International Conference on Acoustics, Speech, and Signal Processing (ICASSP '04), vol. 2, pp. 3986-3990, 2004.

[25] M. Haardt and J. A. Nossek, "Simultaneous schur decomposition of several matrices to achieve automatic pairing in multidimensional harmonic retrieval problem," IEEE Transactions on Signal Processing, vol. 46, no. 1, pp. 161-169, 1998.

[26] R. O. Schmidt, "Multiple emitter location and signal parameter estimation," IEEE Transactions on Antennas and Propagation, vol. 34, no. 3, pp. 276-280, 1986. 


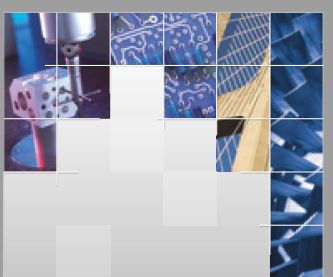

\section{Enfincering}
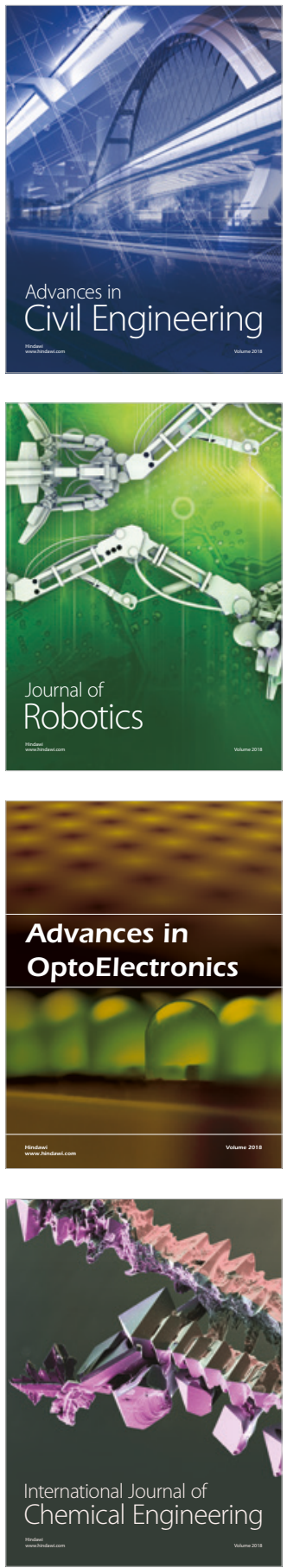

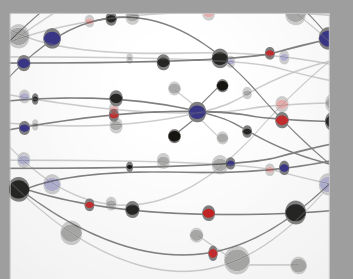

\section{Rotating \\ Machinery}

The Scientific World Journal

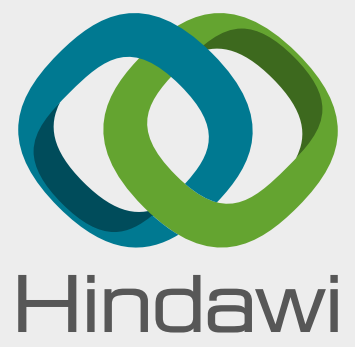

Submit your manuscripts at

www.hindawi.com
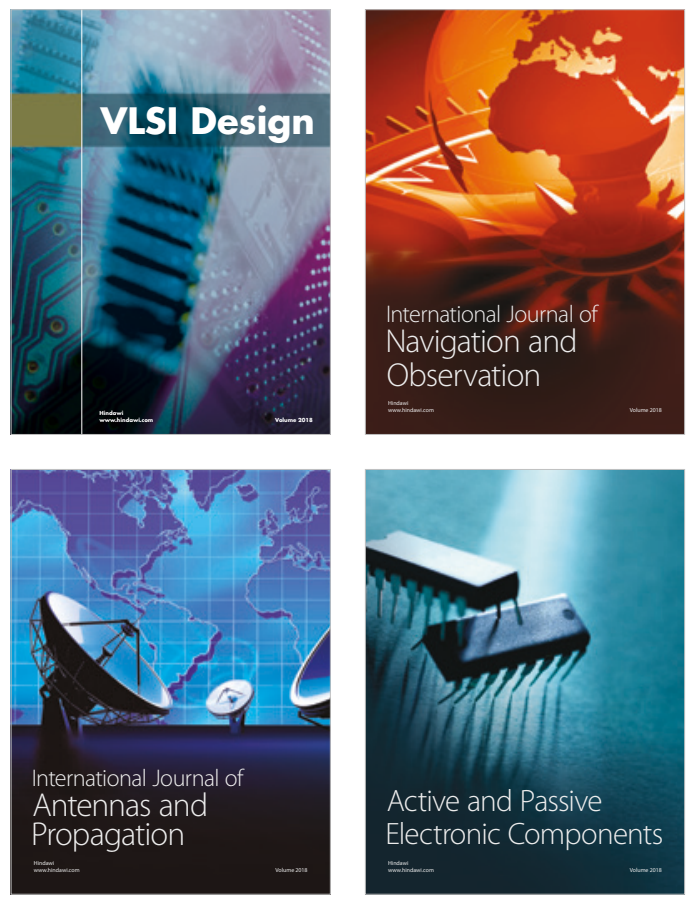
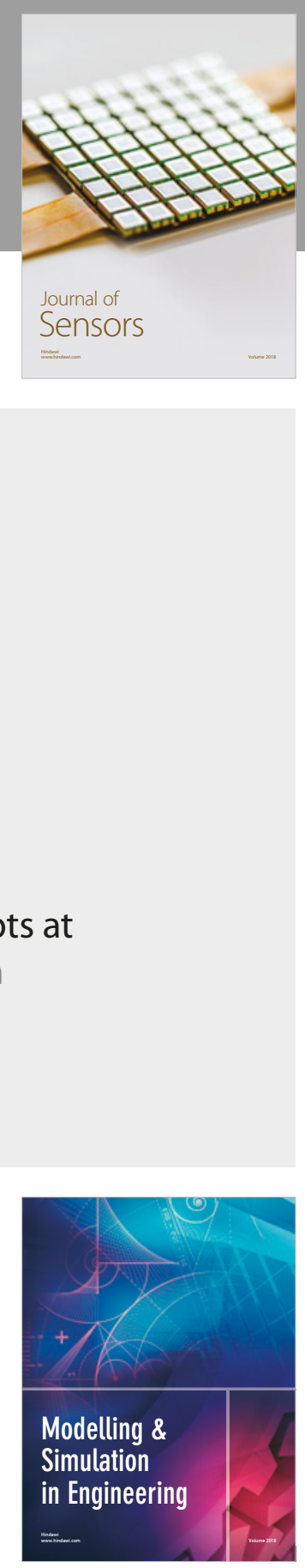

\section{Advances \\ Multimedia}
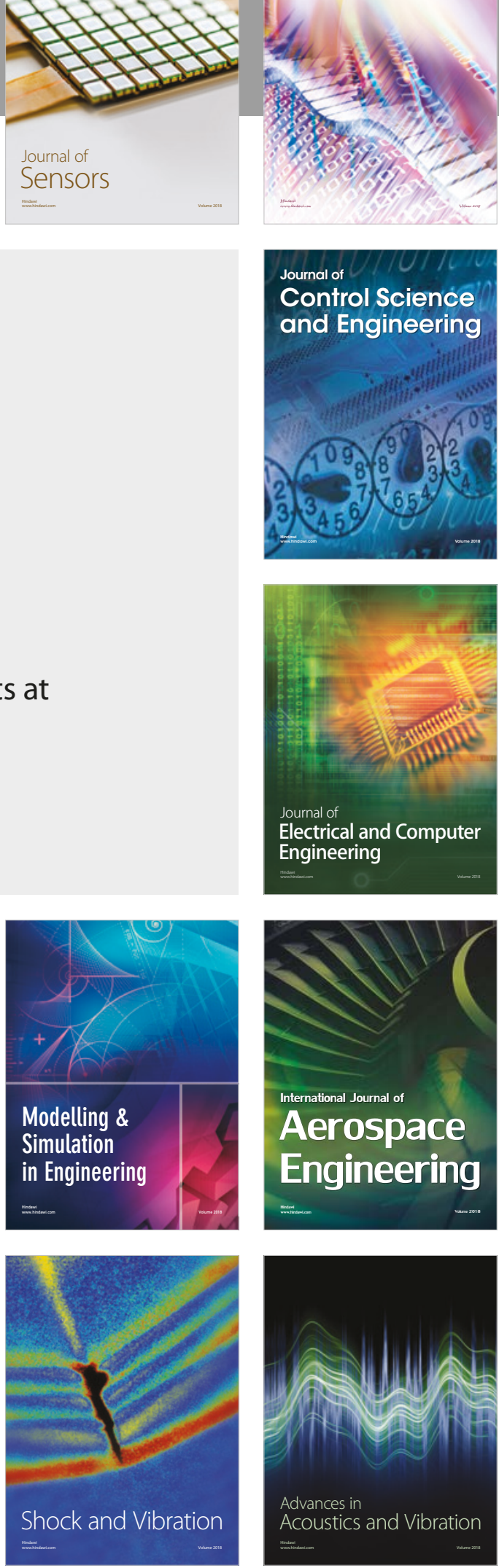